\title{
BEAM-LOSS DRIVEN DESIGN OPTIMIZATION FOR THE SPALLATION NEUTRON SOURCE (SNS) RING*
}

\author{
J. Wei ${ }^{\dagger}$, J. Beebe-Wang, M. Blaskiewicz, P. Cameron, G. Danby, C.J. Gardner, J. Jackson, Y.Y. Lee
} H. Ludewig, N. Malitsky, D. Raparia, N. Tsoupas, W.T. Weng, S.Y. Zhang, BNL, USA

\section{Abstract}

This paper summarizes three-stage design optimization for the Spallation Neutron Source (SNS) ring: linear machine design (lattice, aperture, injection, magnet field errors and misalignment), beam core manipulation (painting, space charge, instabilities, RF requirements), and beam halo consideration (collimation, envelope variation, e-p issues etc).

\section{INTRODUCTION}

The SNS ring compresses $2 \times 10^{14}$ protons at $1 \mathrm{GeV}$ into a $1 \mu \mathrm{s}$ pulse at a repetition rate of $60 \mathrm{~Hz}$ providing a beam power of $2 \mathrm{MW}$ [1]. At such intensity and power, beam loss is a critical issue. Four levels of loss control are implemented: to guarantee hands-on maintenance (average $1 \mathrm{~W} / \mathrm{m}$ [2]), the total uncontrolled beam loss in the ring needs to be limited well below $10^{-3}$; the collimators are designed to collect beam halo at a level of $10^{-3}$; hardware and shielding are designed to withstand $10^{-2}$ for engineering reliability; and the machine can withstand a couple of full beam pulses for emergency handling and commissioning.

Presently, concern of beam loss is incorporated in the design of the ring and transfer lines (HEBT, RTBT) at three stages: linear machine design (lattice, aperture, injection and extraction, magnet field errors and misalignment, etc.), beam core manipulation (space charge, painting, instabilities, $\mathrm{RF}$ requirements, etc.), and beam halo consideration (collimation, beam envelope variation, e-p issues, etc.).

\section{LINEAR MACHINE OPTIMIZATION}

\subsection{Lattice and Acceptance Optimization}

The SNS ring lattice is four-fold symmetric with FODO structures in the arc which are flexible for tuning. By lowering the vertical tune [3], the transverse tunes can be separated by 1 unit to minimize the transverse coupling. Reducing the phase advance in the arcs also makes focusing smoother and, consequently, reduces beam envelope variation (by a factor of 2 in $\beta_{\max } / \beta_{\min }$ ). Recent space charge simulation using SIMPSONS [4] indicates that emittance growth and tail development is noticeably reduced.

In order to minimize off-momentum optics mismatch and to improve dynamic acceptance (DA), we proposed a correction scheme using 4 families of arc sextupoles. By reducing the off-momentum beta wave from $\pm 12 \%$ to less than $\pm 3 \%$, the off-momentum DA is increased by as much as $30 \%$ (Fig. 1). The momentum aperture is also increased by about $30 \%$ (Tab. 1).

\footnotetext{
* Work performed under the auspices of the US Department of Energy.
}

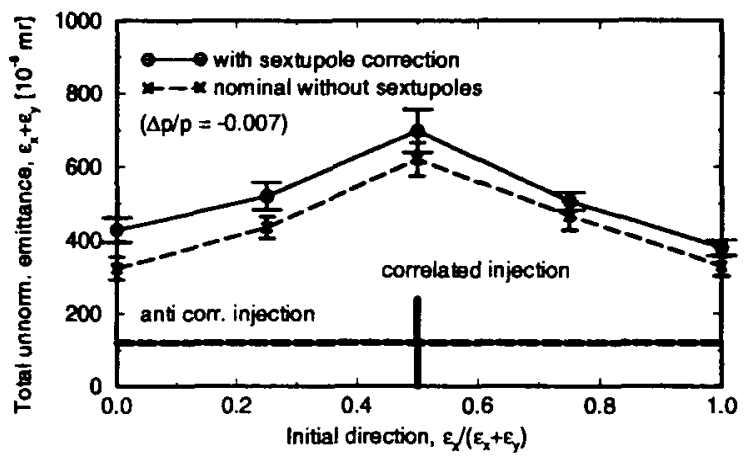

Figure 1: Improvement in the DA due to off-momentum optics optimization using arc sextupoles. The 6 dimensional tracking is done with TEAPOT incorporating expected magnetic errors, misalignments, and physical aperture of the machine with 10 random seeds at 5 transverse initial directions.

Table 1: Momentum aperture and sextupole improvement.

\begin{tabular}{lc}
\hline Item & Value \\
\hline Beam momentum spread (99\%) & \pm 0.007 \\
RF acceptance at $40 \mathrm{kV}$ & \pm 0.010 \\
Ring acceptance at nominal emittance & \pm 0.015 \\
Ring acceptance with sextupole correction & \pm 0.020 \\
\hline
\end{tabular}

In order to decouple injection ramping from lattice tuning, and to accommodate possible future laser-undulator charge exchange injection schemes [5], we designed an alternative hybrid lattice [3] with FODO arcs but doublet straight sections. The long uninterrupted straight section can possibly accommodate the entire injection assembly. Since locations of peak $\beta_{x, y}$ are only at places of large aperture quadrupoles, the horizontal DA is increased by about

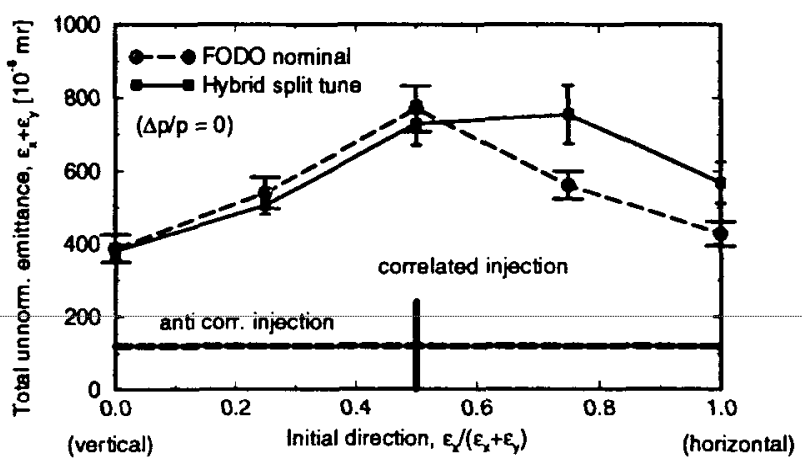

Figure 2: Improvement in the DA with the hybrid lattice. 


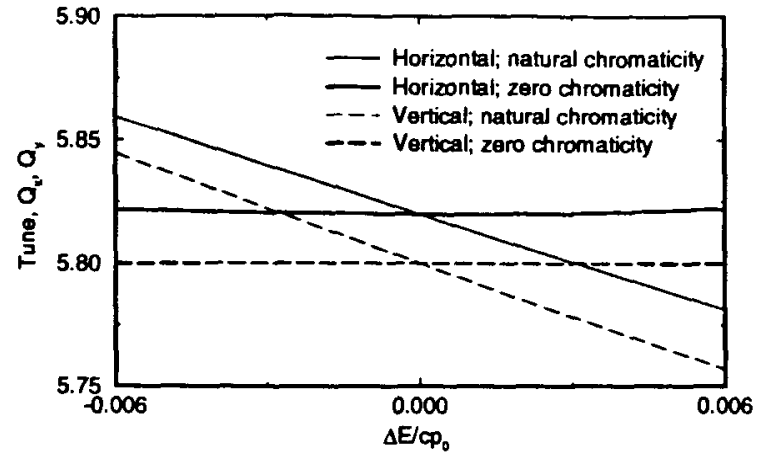

Figure 3: Chromatic tunability with 4-family sextupoles.

30\% (Fig. 2).

\subsection{Chromatic Tunability}

With four families of sextupoles, desired chromatic tune variation can be achieved across the entire range of beam momenta. Without enhancing the nonlinear chromaticity, the linear chromaticity can be either reduced or enhanced for tune spread and DA optimization and for possible instability damping (Fig. 3).

\subsection{Magnetic Errors and Misalignments}

Field errors in both dipoles and quadrupoles (Tab. 2) are designed to be at $10^{-4}$ level within a $92 \%(7.6 \mathrm{~cm})$ of radius to iron pole tip. Dipole ends are optimized to provide sector magnet function to $10^{-4}$ accuracy. Quadrupole ends are optimized to reduce the integrated $b_{6}$ error. With such field quality, the magnet error impact is small. Magnet misalignment (Tab. 3) can cause significant closed orbit offset and coupling requiring designed corrections [6].

Tab. 4 indicates that the leading sources of loss are expected to be incoming LINAC halo, LINAC gap residual, injection loss, and foil scattering. Foil loss is doubled in the anti-correlated painting scheme. At an off-normal condition of doubled LINAC emittance, the loss will again be doubled due to increasing foil hits.

Table 2: Expected magnetic errors of ring quadrupoles based on AGS Booster magnet measurement data and end optimization. Normalized to $10^{-4}$ of the main field at the reference radius $\left(R_{r e f}\right)$ of $7.6 \mathrm{~cm}$. ( $n=2$ is quadrupole).

\begin{tabular}{lrrrr}
\hline$n$ & \multicolumn{2}{c}{ Normal } & \multicolumn{2}{c}{ Skew } \\
& $\left\langle b_{n}\right\rangle$ & $\sigma\left(b_{n}\right)$ & $\left\langle a_{n}\right\rangle$ & $\sigma\left(a_{n}\right)$ \\
\hline Body & [unit] & & & \\
3 & - & -2.46 & - & -2.5 \\
4 & - & -0.76 & - & -2.0 \\
5 & - & -0.63 & - & 1.29 \\
6 & 0.20 & 0.0 & - & 1.45 \\
7 & - & 0.02 & - & 0.25 \\
8 & - & -0.63 & - & 0.31 \\
9 & - & 0.17 & - & -0.11 \\
10 & 0.70 & 0.0 & - & 1.04 \\
14 & -1.41 & 0.0 & - & 0.26 \\
End & [unit.m] & & (Length=0.15 m) \\
6 & 0.25 & - & - & - \\
\hline
\end{tabular}

Table 3: Expected alignment errors of ring magnets.

\begin{tabular}{lr}
\hline Item & Value \\
\hline Integral Transfer Function variation (rms) & $10^{-4}$ \\
Ring dipole sagitta deviation & $3 \mathrm{~cm}$ \\
Magnetic center position (rms) & $0.1 \mathrm{~mm}$ \\
Magnet longitudinal position (rms) & $0.5 \mathrm{~mm}$ \\
Mean field roll angle (ms) & $0.2 \mathrm{mr}$ \\
\hline
\end{tabular}

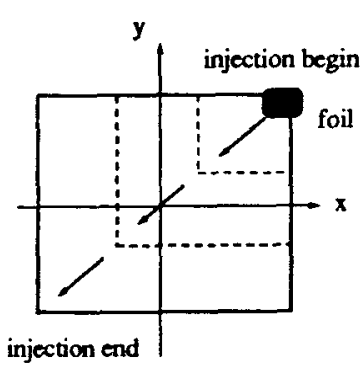

(a)

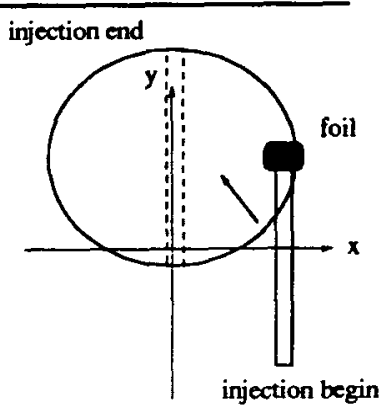

(b)
Figure 4: Correlated and anti-correlated painting of the transverse phase space (physical space shown).

\section{BEAM CORE MANIPULATION}

\section{I Injection Painting and RF Gymnastics}

The beam phase space distribution is manipulated [7] during $\mathrm{H}^{-}$injection both to achieve desired beam distribution at the target and to maintain the space charge tune shift at a moderate level $(\sim 0.2)$. The beam is injected at a location of near-zero dispersion to avoid complications caused by transverse-longitudinal coupling. In the longitudinal direction, the frequency of the pre-injection transfer line $\mathrm{RF}$ system is modulated to broaden the beam momentum spread without enhancing the momentum tail. In the transverse direction, both correlated and anti-correlated painting schemes (Fig. 4) can be accommodated to optimize operation. The anti-correlated scheme minimizes the impact of transverse coupling produced by misalignment and space charge forces, while the correlated scheme reduces halo development by gradually painting to larger action.

Table 4: SNS ring loss budget at $2 \mathrm{MW}$ power assuming $400 \mu \mathrm{g} / \mathrm{cm}^{2}$ foil density with the anti-correlated painting.

\begin{tabular}{lll}
\hline Mechanism & Controlled loss & Uncontrol. loss \\
\hline LINAC incoming halo & & $10^{-4}$ \\
$\begin{array}{l}\text { LINAC gap residual } \\
\text { Injection loss: }\end{array}$ & $10^{-5}$ \\
\hline missing foil & 0.12 & - \\
foil elastic scattering & - & $5.2 \times^{-5}$ \\
foil inelastic scattering & - & $3.7 \times 10^{-5}$ \\
$\mathrm{H}^{0}$ production & $0.02 \sim 0.11$ & $2 \sim 11 \times 10^{-8}$ \\
ionization energy loss & - & $5 \times 10^{-6}$ \\
beam hitting septum & $<10^{-3}$ & \\
Ring collimator & $\sim 10^{-3}$ & \\
Fault condition: & & \\
\hline I kicker missing & $<0.1$ & - \\
LINAC off-normal: & & \\
\multicolumn{1}{l}{ doubled emittance } & $0.03 \sim 0.11$ & $2.1 \times 10^{-4}$ \\
\hline
\end{tabular}




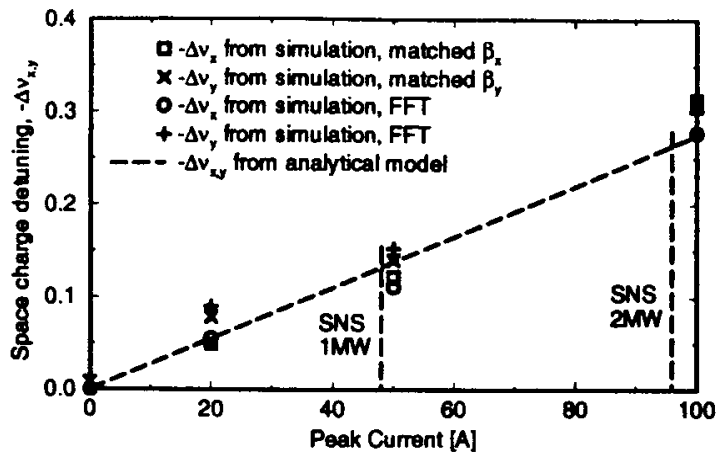

Figure 5: Benchmarking of SIMPSONS simulation.

\subsection{Space Charge Simulation}

Files prepared by TEAPOT $[8,9]$ containing magnetic field errors, misalignments, and corrections are used for SIMPSONS [4] space charge simulation (Fig. 5).

\section{BEAM HALO CONSIDERATION}

Incoming beam halo from the LINAC is collimated transversely at the first two FODO cells of the transfer line (HEBT) using movable foil stripping and collimator/dump combination, and longitudinally at the maximum dispersion location of the $90^{\circ}$ achromat bend in the HEBT.

\section{I Two-Stage Ring Collimation}

Ring collimation employs two-stage system located in a shielded non-dispersive straight section. The movable primary collimator (Fig. 6) can be used both to intercept incoming halo and for beam diagnosis. Three secondary collimators located around $220 \pi \mathrm{mm} \cdot \mathrm{mr}$ acceptance at phases $\left(\phi_{1}, \phi_{2}, \phi_{3}\right)$ determined from efficiency optimization and lattice constraints, are designed to catch scattered particles.

\subsection{Surface Grazing}

The Collimators consists of layers of steel, borated water, etc to contain charged and uncharged secondary particles $[10,11]$. Coated, saw-toothed surface is studied to minimize secondary electron emission, as confirmed in a recent experiment at the Tandem [12]. Surface preparation together with two-stage collimation also minimizes beam loss caused by grazing scattering (Tab. 5).

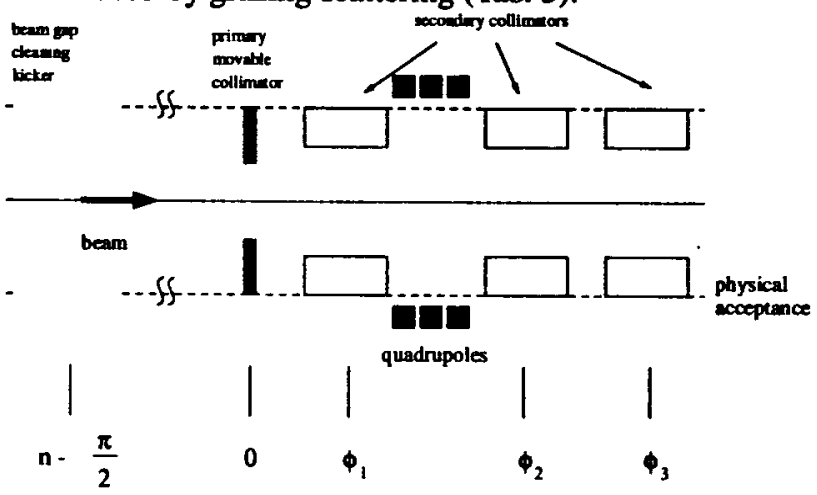

Figure 6: Schematic layout of SNS ring beam gap cleaning and collimation devices. The collimators are housed and shielded in a single straight section.
Table 5: Monte-Carlo simulation of proton grazing showing probability of escape, median escape angle, median energy loss, and correlation coefficient of scattered proton as functions of incident angle.

\begin{tabular}{lcccc}
\hline $\begin{array}{l}\text { Angle } \\
\text { [mr] }\end{array}$ & $\begin{array}{c}\text { Prob. of } \\
\text { escape }\end{array}$ & $\begin{array}{c}\text { Med. esc. } \\
\text { angle [mr] }\end{array}$ & $\begin{array}{c}\text { Med. energy } \\
\text { loss [MeV] }\end{array}$ & $\begin{array}{c}\text { Corr. } \\
\text { coeff. }\end{array}$ \\
\hline 0.1 & 0.96 & 0.3 & 0.05 & 0.9 \\
1 & 0.84 & 3.2 & 4.4 & 0.9 \\
5 & 0.67 & 12 & 60 & 0.8 \\
10 & 0.54 & 20 & 153 & 0.8 \\
15 & 0.45 & 28 & 239 & 0.7 \\
20 & 0.37 & 35 & 314 & 0.7 \\
25 & 0.30 & 41 & 369 & 0.7 \\
\hline
\end{tabular}

\subsection{Beam Gap Cleaning}

Chopper inefficiency, foil ionization, etc. can produce residual beam between subsequent micro bunches resulting in uncontrolled loss at extraction. A gap cleaning kicker is designed to be located at a straight section with a vertical betatron phase $90^{\circ}$ (modulus integer) from that of the primary collimator, where beam loss is measured with a fast gated loss monitor [13]. The hardware is similar to that of the RHIC Damper/Tune Monitor System, which uses commercially available MOSFET banks to supply $5 \mathrm{kV}, 120 \mathrm{~A}$, $10 \mathrm{~ns}$ rise and fall time pulses to a transmission line kicker. Burst mode frequency is greater than $1 \mathrm{MHz}$, permitting turn-by-turn kicking. With 5 meters of kicker length, the gap can be cleaned in about 25 turns.

\subsection{Impedance, Instability, e-p Issues}

We have compiled an impedance budget and started measurement to benchmark impedance model. Based on the budget, instability growth rate is monitored to guide the design. Experimentally, we are studying a newly observed e-p like instability in AGS Booster to understand the mechanism $[14,15]$.

We thank G. Rees for many enlightening discussions, $S$. Machida, R. Talman for codes assistance, and many others including J. Alessi, J. Galambos, A. Jason, A. Luccio, R. Macek, T. Satogata, T. Sen, A. Soukas, S. Tepikian, P. Thieberger, J. Tuozzolo, J.G. Wang, C. Warsop, R. Witkover.

\section{REFERENCES}

[1] Spallation Neutron Source Design Manual, June 1998.

[2] T. Wangler, RF Linear Accelerators, Wiley \& Sons, p. 285.

[3] C.J. Gardner, Y.Y. Lee, N. Tsoupas, J. Wei, PAC99 (1999).

[4] S. Machida, Nucl. Instrum. Methods, A309 (1991) 43.

[5] Y. Suzuki; I. Yamane, ICFA Inj.JExt. Workshop (1999).

[6] C.J. Gardner, Y.Y. Lee, W.T. Weng, EPAC98 (1998).

[7] J. Beebe-Wang, et al, PAC99 (1999).

[8] L. Schachinger, R. Talman, Part. Accel. 22, 35 (1987).

[9] N. Malitsky, J. Smith, J. Wei, PAC99 (1999).

[10] H. Ludewig, et al, PAC99 (1999).

[11] S.Y. Zhang, PAC99 (1999).

[12] P. Thieberger, et al. BNL/SNS 57 (1999).

[13] R. Witkover, et al, BNL/SNS 49 (1998); PAC99 (1999).

[14] J. Wei, et al, ICFA Newsletter No.19 (1999).

[15] M. Blaskiewicz, PAC99 (1999). 